\title{
Previsão da taxa de juros Selic e do câmbio nominal a partir de um modelo Var com restrição
}

\author{
Luciano D'Agostin ${ }^{*}$ \\ José Luís da Costa Oreiro**
}

Os indicadores macroeconômicos de emprego, produto e inflação são os mais importantes para a definição da política monetária e cálculo de juros básicos pelo Federal Reserve (FED). Os juros básicos dos EUA estão para o mundo como a taxa Selic está para o Brasil. Quaisquer alterações nas expectativas em relação à taxa básica de juros dos EUA provocam enormes movimentos nas taxas de câmbio, juros internacionais e no mercado de ativos ao redor do mundo.

A economia norte-americana atravessa uma fase de combate a inflação, aliado ao aumento dos gastos do governo com segurança e recuperação de infra-estruturas de cidades destruídas por catástrofes recentes (furacões), déficits crescentes na conta corrente (superior a $6 \%$ do PIB) e queda de $14,4 \%$ das reservas internacionais desde dezembro de 2004 (US\$ 90 bilhões para US\$ 77 bilhões em setembro de 2005). Como conseqüência, nos últimos meses, o dólar se depreciou, não somente em relação ao real, mas em relação as principais moedas internacionais.

Desde julho de 2004, são observados sucessivos aumentos das taxas de juros dos títulos do Tesouro norte-americano (2,15\% a.a. em julho de 2004 para 4,25\% a.a. em outubro de 2005, ou seja, quase $100 \%$ de alta). Segundo indicação do relatório de política monetária do FED, de setembro de 2005, as taxas de juros americanas seguem tendência de alta por dois motivos: (i) para combater a inflação e; (ii) para captar dinheiro externo para financiar os déficits do governo. De qualquer modo, o mercado financeiro mundial deve continuar muito volátil a cada divulgação de indicadores econômicos dos EUA..

Qual a implicação no curto prazo desta indicação de tendência de alta das taxas de juros norte-americanas para o câmbio nominal dólar/real?

No curto prazo, o Brasil pode ser penalizado pelo maior custo de capital vigente no mercado internacional, ou seja, pode haver migração de uma parcela da massa de dinheiro externo para aquisição de títulos da dívida norte-americana, que ainda apresenta risco baixíssimo pela alta capacidade de pagamento em relação aos títulos públicos do Brasil, apesar

\footnotetext{
* Mestre em Desenvolvimento Econômico pela UFPR. Professor Assistente da Faculdade Radial e do IBPEX. Endereço eletrônico: lucianodagostini@yahoo.com.br.

** Doutor em Economia (IE/UFRJ), Professor do Departamento de Economia da UFPR e pesquisador do CNPq. Endereço eletrônico: joreiro@ufpr.br. Web site: www.joseluisoreiro.ecn.br.
} 
deste também estar com risco baixo. Para um país como o Brasil, que tem amortizações de empréstimos externos na ordem de 40 a 50 bilhões de dólares por ano, o nível mais alto das taxas de juros americanas é fator vital para definir o curso do risco-país e, por conseguinte, as expectativas de crescimento, captação de capital e recursos estrangeiros, câmbio nominal e juros domésticos.

Como estamos falando de uma relação entre duas moedas, a taxa de câmbio nominal dólar/real também é determinada pelos movimentos macroeconômicos da economia brasileira, em especial, pelo balanço de pagamentos e as conduções de política monetária e fiscal.

Por exemplo, uma política monetária menos restritiva (queda da taxas de juros), no curto prazo, aumenta o volume de moeda, o nível de preços e provoca aumento do nível de investimento e produto; enquanto que, pelo lado do balanço de pagamentos, age de forma negativa na conta de capital, pois os agentes econômicos observam o diferencial entre a taxa de juros doméstica e a internacional para determinar seu nível de aplicação no mercado de capitais. Assim, uma queda no diferencial de juros pode fazer com que haja queda de entrada de capital externo no país fazendo, por fim, com que haja pressões para depreciação cambial.

É certo que o câmbio no Brasil é flutuante. Porém, no mês de outubro e novembro, conforme atas do Comitê de Política Monetária (COPOM), o governo fez pequenas intervenções no sentido de conter a rápida apreciação da moeda brasileira, perante a moeda norte americana. Pelos dados do Banco Central do Brasil (BCB), a Taxa de Câmbio Nominal Livre para compra (série 3697 -média de período), caiu quase 5\%, de 2,294 em setembro para 2,187 até meados de novembro.

$\mathrm{O}$ BCB, por intermédio do sistema de Metas de Inflação, tem sob sua responsabilidade manter a taxa de inflação sob controle. Este sistema faz da taxa de juros SELIC o principal instrumento de política monetária para alcançar o objetivo proposto ${ }^{10}$. Por isso a fixação das taxas de juros em cada reunião do COPOM se apóia na previsão de inflação.

Sabe-se que a meta de inflação para 2005 é de 5,1\%, com um teto máximo de 7,6\%. Previsões do BCB estimam o IPCA acumulado para 2005, em 6,36\%. No mês de outubro,

\footnotetext{
${ }_{10}$ Para detalhes ver: D’Agostini, L.; Oreiro, J.L.C. "Prevendo o Comportamento de Curto-Prazo da Taxa de Juros SELIC a Partir de um modelo VAR com Restrição”. Boletim de Conjuntura \& Tecnologia, Ano 01, Vol..02, julho/agosto, 2005.
} 
observou-se o IPCA de $0,75 \%$, superior as estimativas de mercado, que estavam em torno de 0,55 a 0,6\%. Assim, o IPCA acumulado no ano (janeiro a novembro) está em 4,64\%.

Diante do objetivo de manter a inflação sob controle e do cenário da economia americana atual, o que acontecerá com a taxa de câmbio nominal real/dólar? Em outras palavras, o impacto da alta da taxa de juros norte-americana será, nos próximos meses, um fator determinante para reverter o processo de depreciação da moeda americana perante o real? A queda gradual da taxa de juros Selic fará com que a moeda nacional volte a se depreciar?

Neste cenário, pelo modelo de Vetores Auto-Regressivos (VAR), o objetivo desta coluna reside em prever: (i) a taxa de câmbio nominal e; (ii) a taxas de juros SELIC ${ }^{11}$, esperadas para os próximos 2 meses (dezembro e janeiro). Acertar antecipadamente as expectativas cambiais e o valor da taxa de juros Selic pode ajudar os agentes econômicos a tomar decisões corretas de investimento e consumo.

No exercício de estimação da Selic, esta será determinada com base na avaliação dos valores correntes e futuros de variáveis da economia brasileira como produto, moeda, câmbio e preços. No exercício de estimação da taxa de câmbio nominal, incluiremos a taxa de juros americana no modelo. Portanto, para a previsão da Selic utilizamos 5 variáveis e para a previsão da taxa de câmbio nominal utilizamos 6 variáveis.

Assumindo endogeneidade em todas as variáveis inseridas no sistema, a taxa de juros de curto prazo e taxa de câmbio nominal pode ser estimada consistentemente pelo VAR. Os procedimentos econométricos usados são resumidos nas seguintes etapas ${ }^{12}$ : (i) Logaritimizar as séries; (ii) Efetuar o teste de raiz unitária pelo ADF; (iii) Efetuar os testes de Cointegração JJ; (iv) Escolher a defasagem ótima do VAR pelo critério de Akaike (AIC) e Schwartz (SBC); (v) Conforme o teste Johansen-Juselius (JJ) adota-se o modelo VAR simples ou com correção de erro (VECM); (vi) determinar os coeficientes das equações por MQO; (vii) observar a equação de equilíbrio de longo prazo entre as variáveis, dado pelo VECM; (viii) substituir os valores presentes e passados na equação da taxa de câmbio e na equação da taxa de juros.

\footnotetext{
11 O modelo VAR, proposto no boletim Economia e Tecnologia (Ano 1, Vol. 02, pág. 29-32) foi muito preciso para prever a taxa SELIC para agosto e setembro de 2005. Por isso, para este artigo, resolveu-se, além de continuar a previsão da SELIC, iniciar o processo de previsão de câmbio nominal.

12 Para detalhes dos procedimentos metodológicos, ver D’Agostini (2004). Monismo Versus Não Monismo no Brasil (1994-2004): Uma Abordagem Econométrica por Vetores Auto-Regressivos com Restrição. Dissertação de Mestrado, nov.2004, 143p, Curitiba: UFPR.
} 
Assim, o exercício na tentativa de previsão da taxa de juros Selic e taxa de câmbio nominal usa dados mensais ${ }^{13}$, de janeiro de 1999 a outubro de 2005. As variáveis são: preços, juros, produto, câmbio e moeda. Para preços, utilizou-se o IPCA, código 433 do IBGE, por ser a taxa de inflação utilizada para estabelecer a Meta de Inflação anual. Para a taxa básica nominal de juros, utilizou-se a Selic, código 4189 do BCB. Esta taxa de juros é a taxa pelo qual o BCB tenta controlar a inflação. Para produto utiliza-se o PIB mensal em valores correntes, medida em milhões de reais, código 4380 do BCB. A série de moeda é $\mathrm{M}_{2}$, código 1837 e a série de câmbio nominal é a 3697, ambos do BCB. Por fim, a taxa de juros americana é Taxa de desconto do Banco Central dos Estados Unidos (FED).

Os resultados da previsão da taxa de juros estimada a partir de um modelo VAR (5) com correção de erros, estão dispostos na Tabela 1, enquanto que a previsão da taxa de câmbio nominal, estimado a partir de um VAR (5) com correção de erros está disposta na Tabela 2.

Tabela 1. Comparação da Taxa Selic efetiva com a taxa Selic estimada em \% a.a.

\begin{tabular}{cccc}
\hline Mês & SELIC Efetiva \% a.a. & Previsão SELIC pelo VAR & Elasticidade Juros \\
\hline Set $/ 05$ & $19,25 \%$ & - & -0.0196229080162 \\
Out $/ 05$ & $19 \%$ & $18,88 \%$ & -0.0195684805901 \\
Nov $/ 05$ & $18,5 \%$ & $18,62 \%$ & -0.0206557234805 \\
Dez $/ 05$ & $?$ & $18,24 \%$ & -0.0270424279011 \\
Jan $/ 06$ & $?$ & $18,06 \%$ & - \\
\hline
\end{tabular}

Fonte: Banco Central do Brasil e Dados da Pesquisa.

Tabela 2. Previsão da Taxa de Câmbio Nominal (US\$/R\$) pelo VAR

\begin{tabular}{cccc}
\hline Mês & Câmbio Efetivo & Previsão Câmbio Nominal** & Elasticidade Câmbio \\
\hline Out/05 & 2,2557 & - & -0.0367853671490 \\
Nov/05 & $2,1871^{*}$ & 2,1727 & -0.012782219075 \\
Dez/05 & $?$ & 2,1449 & 0.00599404705023 \\
Jan/06 & $?$ & 2,1578 & -0.018253043257 \\
Fev/06 & $?$ & 2,1184 & -
\end{tabular}

Fonte: Banco Central do Brasil e Dados da Pesquisa.

Nota: * 2,1871 corresponde a média do câmbio nominal nos primeiros 16 dias de novembro.

**A margem de erro da previsão é de $2 \%$.

13 Os dados foram extraídos dos endereços eletrônicos do Banco Central do Brasil (BCB), Federal Reserve Bank (FED) e Fundo Monetário Internacional (FMI).

42 
Os resultados da Tabela 1 e 2 mostram uma provável queda de 0.25 pontos percentuais na taxa de juros SELIC na próxima reunião do COPOM para o mês de dezembro. No que se refere à taxa de câmbio nominal, o modelo prevê que ela permanecerá abaixo de $\mathrm{R} \$ 2,20$ nos próximos meses.

O movimento da SELIC é resultado do bom comportamento do IPCA dentro do sistema de Metas de Inflação. Assim, a queda da taxa de juros é o único resultado esperado do modelo. Com base na Tabela 1 , percebemos que as elasticidades negativas dos juros com respeito às demais variáveis do sistema, calculadas pelo modelo, nos meses de setembro a dezembro, sinalizam a continuidade do movimento de redução da taxa de juros SELIC, iniciada no mês de setembro.

Para o mês de dezembro o modelo indica a previsão da SELIC em 18,24\%, pois a elasticidade juros do mês de novembro é - 0.0206557234805. Neste caso, a taxa cairia dos atuais $18,5 \%$ ao ano para $18,25 \%$, diferentemente da taxa de $18 \%$ ao ano esperada pela maior parte dos analistas. Do mesmo modo, pela análise da elasticidade juros, a previsão da SELIC para o mês de janeiro é de 18,06\%, o que indica uma queda de 0,25 ponto percentual .

No mais, o resultado da taxa de câmbio nominal, mesmo com a previsão de queda da SELIC, mostra que o real continuará o movimento de valorização perante o dólar. Para novembro a previsão do câmbio é US $\$ 1,00=\mathrm{R} \$ 2,17$; para dezembro, $\mathrm{R} \$ 2,14$, para janeiro $\mathrm{R} \$ 2,15$ e para fevereiro $\mathrm{R} \$ 2,11$. Assim, entre outubro e fevereiro espera-se uma queda do câmbio nominal em torno de 6,09\%. 


\section{ANEXO}

A equação (1) de previsão da taxa de juros simulada é:

$\mathrm{D}($ LOGSELIC $)=-0.02294421295 *($ LOGSELIC $(-1)-0.03418650552 *$ LOGM2(-1) + 1.451277395*LOGCAMBIO(-1) - 0.9844383958*LOGIPCA(-1) - 2.668805715*LOGPIB(1) + 0.01845294899*(@TREND(99:01)) + 26.05981922) + 0.4669404645*D(LOGSELIC(1)) - $0.07714116023 * \mathrm{D}(\operatorname{LOGSELIC}(-2))+0.08818968947 * \mathrm{D}(\operatorname{LOGSELIC}(-3))$ $0.08630174673 * \mathrm{D}($ LOGSELIC $(-4))+\quad+\quad 0.02445978526 * \mathrm{D}(\operatorname{LOGSELIC}(-5))$ $0.1198580932 * \mathrm{D}($ LOGM2(-1)) $\quad+\quad 0.01679441881 * \mathrm{D}(\operatorname{LOGM} 2(-2))$ $0.157503198 * \mathrm{D}($ LOGM2(-3)) $\quad+\quad 0.3731258339 * \mathrm{D}(\operatorname{LOGM} 2(-4))$ $0.2822926306 * \mathrm{D}(\operatorname{LOGM} 2(-5))+0.1171188923 * \mathrm{D}(\operatorname{LOGCAMBIO}(-1))$ $0.02270091733 * \mathrm{D}(\mathrm{LOGCAMBIO}(-2))+0.1233151027 * \mathrm{D}(\mathrm{LOGCAMBIO}(-3))$ $0.03522360392 * \mathrm{D}(\operatorname{LOGCAMBIO}(-4))+0.1444976448 * \mathrm{D}(\operatorname{LOGCAMBIO}(-5))$ $0.01965267001 * \mathrm{D}(\operatorname{LOGIPCA}(-1)) \quad-\quad 0.009820948139 * \mathrm{D}(\mathrm{LOGIPCA}(-2))$ $0.009494804641 * \mathrm{D}(\mathrm{LOGIPCA}(-3)) \quad-\quad 0.005562892631 * \mathrm{D}(\mathrm{LOGIPCA}(-4))$ $0.00396533348 * \mathrm{D}(\operatorname{LOGIPCA}(-5)) \quad-\quad 0.01477243062 * \mathrm{D}(\operatorname{LOGPIB}(-1))$ $0.03555903298 * \mathrm{D}(\mathrm{LOGPIB}(-2))+0.0320856195 * \mathrm{D}(\mathrm{LOGPIB}(-3))$ $0.08059311319 * \mathrm{D}(\operatorname{LOGPIB}(-4)) \quad-\quad 0.05532461271 * \mathrm{D}(\operatorname{LOGPIB}(-5)) \quad-0.01026044214 \quad+$ 6.895524101e-05*(@TREND(99:01))

A equação (2) de previsão da taxa de câmbio nominal simulada é:

$\mathrm{D}(\mathrm{LOGCAMBIO})=-0.194488751 *($ LOGCAMBIO $(-1)-10.32433446 *$ LOGPIB $(-$ 1) - 0.7491373393*LOGIPCA(-1) + 0.09576390462*(@TREND(99:01)) + 114.7546803 ) 0.05511767255*( LOGJUROSEUA(-1) + 20.36288743*LOGPIB $(-1)+$ 2.20356983*LOGIPCA(-1) - 0.1943025364*(@TREND(99:01)) - 228.4163122 ) + $0.01575168997 *(\quad$ LOGSELIC $(-1)+10.92320841 *$ LOGPIB $(-1)$ 0.04428625344*LOGIPCA(-1) - 0.106948039*(@TREND(99:01)) - 125.6257224 ) 0.3314721353*(LOGM2(-1) + 3.896140762*LOGPIB(-1) + 0.08848188877*LOGIPCA(-1) 0.04868016423*(@TREND(99:01)) - 62.904981 ) + 0.3952783024*D(LOGCAMBIO(-1)) + $0.2009054755^{*} \mathrm{D}(\mathrm{LOGCAMBIO}(-2))+0.2729411247 * \mathrm{D}(\operatorname{LOGCAMBIO}(-3))+$ $0.1719491951 * \mathrm{D}(\mathrm{LOGCAMBIO}(-4))+0.1887203433 * \mathrm{D}(\operatorname{LOGCAMBIO}(-5))+$ $0.1135991374 * \mathrm{D}($ LOGJUROSEUA(-1)) $\quad+0.04940261779 * \mathrm{D}($ LOGJUROSEUA(-2) $)+$ $0.02532161574 * \mathrm{D}($ LOGJUROSEUA $(-3)) \quad+0.04545814464 * \mathrm{D}($ LOGJUROSEUA(-4)) $\quad+$ $0.05694945024 * \mathrm{D}(\mathrm{LOGJUROSEUA}(-5)) \quad-\quad 0.1603153436 * \mathrm{D}(\mathrm{LOGSELIC}(-1)) \quad+$ $0.2347005827 * \mathrm{D}($ LOGSELIC(-2)) - $\quad 0.2076439967 * \mathrm{D}(\operatorname{LOGSELIC}(-3))$ $0.07343805058 * \mathrm{D}($ LOGSELIC $(-4))+0.07319624791 * \mathrm{D}($ LOGSELIC $(-5)) \quad-$ $0.1566056153 * \mathrm{D}($ LOGM2(-1)) $+0.2589693836 * \mathrm{D}(\mathrm{LOGM} 2(-2))-0.251722842 * \mathrm{D}$ (LOGM2(3)) - $\quad 0.0551996551 * \mathrm{D}(\mathrm{LOGM} 2(-4))$ $0.2472109435 * \mathrm{D}(\operatorname{LOGPIB}(-1)) \quad+$ $0.1027631343 * \mathrm{D}(\mathrm{LOGPIB}(-3))$ $\begin{array}{llll}0.05310217099 * \mathrm{D}(\operatorname{LOGPIB}(-5)) & + & 0.000139042711 * \mathrm{D}(\operatorname{LOGIPCA}(-1)) & - \\ 0.004484352271 * \mathrm{D}(\operatorname{LOGIPCA}(-2)) & + & 0.004822000427 * \mathrm{D}(\operatorname{LOGIPCA}(-3)) & -\end{array}$ $0.004570432236 * \mathrm{D}($ LOGIPCA $(-4))+0.004177308828 * \mathrm{D}($ LOGIPCA $(-5))+0.002296041625$ -7.093899229e-05*(@TREND(99:01)) 
Onde $\mathrm{D}($.$) é a primeira diferença; (-1),(-2) \ldots,(-5)$ são as defasagens das variáveis utilizadas no modelo e; @TREND (.) é o componente de tendência.

Os modelos VAR são do tipo LOG-LOG. Isto faz com que os coeficientes dos parâmetros estimados sejam interpretados como elasticidades da taxa de juros com respeito às variáveis do sistema, na equação 1 , e elasticidades da taxa de câmbio nominal com respeito às variáveis do sistema, na equação 2 .

Efetuando as substituições dos valores das variáveis defasadas no sistema, para diversos períodos de tempo, temos o valor das elasticidades dos juros e do câmbio. Estas elasticidades por sua vez determinam, para os períodos $t+1, t+2, \ldots, t+n$, os valores das previsões das taxas de juros subseqüentes.

Por questões probabilísticas do modelo, quanto maior é o tempo de previsão menor é a possibilidade de acerto. Portanto, o modelo VAR serve apenas para previsões de curto prazo. Por isso, nas Tabelas 1 e 2, mostra-se os valores esperados da SELIC e câmbio nominal para três períodos à frente, ou seja, de dezembro a fevereiro. 
